\title{
Transfusion outcomes in patients undergoing coronary artery bypass grafting treated with prasugrel or clopidogrel: TRITON-TIMI 38 retrospective data analysis
}

\author{
Lawrence T. Goodnough, MD, ${ }^{\mathrm{a}}$ Peter K. Smith, MD, ${ }^{\mathrm{b}}$ Jerrold H. Levy, MD, ${ }^{\mathrm{c}}$ Robert S. Poston, MD, ${ }^{\mathrm{d}}$ \\ Mary A. Short, MSN, ${ }^{e}$ Govinda J. Weerakkody, PhD, ${ }^{\mathrm{e}}$ and LeRoy A. LeNarz, MD
}

\begin{abstract}
Objective: Coronary artery bypass grafting-related bleeding and associated transfusion is a concern with dual antiplatelet therapy in patients with acute coronary syndromes. The objective of the present study was to characterize a potential risk-adjusted difference in transfusion requirements between prasugrel and clopidogrel cohorts.

Methods: The data from 422 patients undergoing isolated coronary artery bypass grafting from the TRial to assess Improvement in Therapeutic Outcomes by optimizing platelet InhibitioN with prasugrel Thrombolysis In Myocardial Infarction 38 were analyzed retrospectively.

Results: We found no difference in baseline transfusion risk scores between cohorts. As predicted, the number of units of red blood cells transfused perioperatively correlated with the transfusion risk score $(P<.0001)$. Overall, the 12-hour chest tube drainage volumes and platelet transfusion rates in the prasugrel cohort were significantly greater. However, no statistically significant differences were found in the number of red blood cell transfusions, total hemostatic components transfused, or total blood donor exposure. A significantly greater number of platelet units were transfused postoperatively in the prasugrel patients who underwent surgery within 5 days or less after withdrawal of drug. In an analysis adjusted for the predicted risk of mortality, total donor exposure was not associated with increased mortality.
\end{abstract}

Conclusions: The use of prasugrel compared with clopidogrel was associated with greater 12-hour chest tube drainage volumes and platelet transfusion rates but without any significant differences in red blood cell transfusions, total hemostatic components transfused, or total blood donor exposure. (J Thorac Cardiovasc Surg 2013;145:1077-82)

\section{Supplemental material is available online.}

Dual antiplatelet therapy with aspirin and a thienopyridine has been shown to prevent thrombotic complications in the setting of acute coronary syndromes (ACS) and percutaneous coronary intervention (PCI) but has also been associated with a risk of major bleeding. ${ }^{1}$ In patients with

\footnotetext{
From the Departments of Pathology and Medicine, ${ }^{\text {a }}$ Stanford University School of Medicine, Stanford, Calif; Division of Thoracic and Cardiovascular Surgery, ${ }^{b}$ Duke University Medical Center, Durham, NC; Department of Anesthesiology, Emory University School of Medicine, Atlanta, Ga; Division of Cardiothoracic Surgery, ${ }^{\mathrm{d}}$ Department of Surgery, University of Arizona School of Medicine, Tucson, Ariz; and Lilly USA, ${ }^{\mathrm{e}}$ LLC, Indianapolis, Ind.

This work was sponsored by Daiichi Sankyo, Inc, Parsippany, NJ, and Eli Lilly and Co, Indianapolis, Ind, and is related to study protocol H7T-MC-TAAL, listed on ClinicalTrials.gov (NCT00097591). Dr Smith was supported by National Heart, Lung, and Blood Institute (grant U01-HL088953).

Disclosures: Authors have nothing to disclose with regard to commercial support.

Received for publication Jan 18, 2012; revisions received July 9, 2012; accepted for publication July 26, 2012; available ahead of print Sept 19, 2012.

Address for reprints: Lawrence T. Goodnough, MD, Departments of Pathology and Medicine, Stanford University School of Medicine, 300 Pasteur Dr, Rm H-1402, M/C 5626, Stanford, CA 94305-5626 (E-mail: Ltgoodno@stanford.edu). $0022-5223 / \$ 36.00$

Copyright (c) 2013 by The American Association for Thoracic Surgery http://dx.doi.org/10.1016/j.jtcvs.2012.07.059
}

ACS with scheduled PCI, prasugrel therapy was found to be associated in the Trial to Assess Improvement in Therapeutic Outcomes by Optimizing Platelet Inhibition With Prasugrel Thrombolysis in Myocardial Infarction 38 (TRITON-TIMI 38) with significantly reduced rates of ischemic events, including stent thrombosis, compared with clopidogrel therapy but with an increased risk of major bleeding, including fatal bleeding. ${ }^{2}$ The key safety endpoints in the TRITON-TIMI 38 study were TIMI major bleeding not related to coronary artery bypass grafting (CABG), non-CABG-related TIMI life-threatening bleeding, and TIMI major or minor bleeding, as defined previously. ${ }^{3}$ In TRITON-TIMI 38, major bleeding not related to $\mathrm{CABG}$ occurred significantly more often in the prasugrel- than in the clopidogrel-treated patients $(2.4 \%$ vs $1.8 \%$, respectively). ${ }^{2}$

To further characterize the relative risks of bleeding between prasugrel and clopidogrel therapy, we undertook an analysis of the relationship among antiplatelet therapy, bleeding, and transfusion outcomes in the cohort of patients who underwent CABG during participation in the TRITONTIMI 38 study. In this clinical setting, the timing of thienopyridine withdrawal before CABG could be an important risk factor for subsequent perioperative bleeding and blood component therapy support. Therefore, we conducted 


$\begin{array}{ll}\text { Abbreviations and Acronyms } \\ \text { ACS } & \text { acute coronary syndromes } \\ \text { ADP } & \text { adenosine diphosphate } \\ = & \text { coronary artery bypass } \\ \text { CABG } & \text { grafting } \\ & =\text { European System for Cardiac } \\ & \text { Operative Risk Evaluation } \\ & \text { Score } \\ = & \text { percutaneous coronary } \\ & \text { intervention } \\ = & \text { red blood cells } \\ \text { PCI } & \text { Society of Thoracic Surgeons } \\ & \text { total donor exposure } \\ \text { RBCs } & \text { total hemostatic components } \\ \text { STS } & \text { Trial to Assess Improvement } \\ \text { TDE } & \text { in Therapeutic Outcomes by } \\ \text { THCs } & \text { Optimizing Platelet Inhibition } \\ \text { TRITON-TIMI } 38 & \text { With Prasugrel Thrombolysis } \\ & \text { in Myocardial Infarction } 38 \\ & \end{array}$

a subgroup analysis by grouping CABG patients according to the number of days from the last dose of the study drug.

\section{METHODS}

The study design and principal results from the previous TRITON-TIMI 38 trial have been published. ${ }^{2,3}$ In brief, TRITON-TIMI 38 was a phase 3 , randomized, double-blind, head-to-head clinical trial comparing the effects of prasugrel versus clopidogrel in patients with ACS who were treated with PCI. $^{2}$ The study enrolled 13,608 subjects at 707 sites in 30 countries from November 2004 to January of 2007 . The patients were randomized to receive a loading dose of $60 \mathrm{mg}$ prasugrel or $300 \mathrm{mg}$ clopidogrel, followed by a daily maintenance dose of $10 \mathrm{mg}$ prasugrel or $75 \mathrm{mg}$ clopidogrel combined with aspirin (75-325 mg/day) for up to 15 months. ${ }^{4}$ Key exclusion criteria included an increased risk of bleeding, the receipt of any thienopyridine within 5 days of randomization, recent thrombolytic therapy, and a history of pathologic intracranial findings or clinical findings considered by the investigator to be associated with an increased risk of bleeding. The primary endpoint was the combined incidence of cardiovascular death, nonfatal myocardial infarction, or nonfatal stroke during at least 12 months after PCI. Safety was assessed using the TIMI bleeding criteria and the following criteria: a decrease in hemoglobin and the occurrence of intracranial hemorrhage.

Additional data collection for the present study, obtained by chart review and limited to the information captured during the subject's study participation using a supplementary case report form, was conducted with the acknowledgement or approval of the ethics committee or regulatory board, as required by local regulations. The TRITON-TIMI 38 publications committee approved the conduct of the present analysis and reviewed the publication before submission. The ClinicalTrials.gov identifier was NCT00097591.

\section{Characterization of Treatment Differences and Statistical Analysis}

The present study analyzed additional data to allow characterization of the differences in perioperative bleeding and transfusion requirements between prasugrel and clopidogrel cohorts as potentially related to the timing of thienopyridine withdrawal before CABG. The use of blood products, including the mean number of units of red blood cells (RBCs), including packed RBC and whole blood, platelets, plasma, and cryoprecipitate, as well as total hemostatic components (THCs included platelets, plasma, and cryoprecipitate) and total donor exposure (TDE including total units of all blood products transfused [ie, RBCs, platelets, plasma, and cryoprecipitate]), were compared by treatment group using the Kruskal-Wallis test. Chest tube bleeding was recorded until chest tube discontinuation.

Transfusion requirements, as potentially related to the timing of thienopyridine withdrawal before CABG were evaluated intra- and postoperatively at 0 to 5 days, 6 to 7 days, and more than 7 days after the last dose of study drug. The Kruskal-Wallis test was used to compare the mean number of RBC and platelet units transfused from the day of the last dose of study drug to $\mathrm{CABG}$ and to compare the average cumulative chest tube blood loss at 12 hours after CABG. Pearson's $\chi^{2}$ test was used to compare the proportion of patients treated with antifibrinolytic agents. The relationship between THC or TDE and the predicted transfusion risk score and the association between total chest tube blood loss and THC or TDE were evaluated using Pearson's correlation coefficient.

\section{Transfusion Risk Score and Predicted Probability of Mortality}

A previously reported and validated transfusion risk score, ${ }^{5}$ calculated using preoperative variables, was used to identify the relationship between the predicted risk of transfusion for each treatment group and the subsequent number of blood components transfused.

To provide a model that included risk adjustment, the predicted probability of mortality for each patient was calculated using the widely accepted and validated Society of Thoracic Surgeons (STS) score ${ }^{6}$ and European System for Cardiac Operative Risk Evaluation Score (euroSCORE). ${ }^{7}$ For the purpose of calculating the transfusion risk score, STS, and euroSCORE, any missing values for risk were considered an absence of risk.

The influence of TDE on the risk of mortality and a comparison of the risk of all-cause death between prasugrel and clopidogrel was evaluated using logistic regression analysis with study drug as a categorical variable and predicted probability of mortality as a covariate.

\section{RESULTS \\ Study Population}

The present retrospective analysis included 485 subjects who underwent $\mathrm{CABG}$ at some point during participation in the 15-month TRITON-TIMI 38 trial. A supplementary case report form could not be obtained for 36 of the 485 patients $(\mathrm{n}=449)$. The type of CABG procedure (isolated or major cardiac procedures in addition to the $\mathrm{CABG}$ procedure) could not be determined in 3 patients $(n=446)$, and 2 patients who died were included in the analysis without the supplementary case report forms $(n=448)$, because mortality was reported for all subjects in the TRITON-TIMI 38 study. The cohort of 448 patients included all those who died and all but 2 patients who were classified in the TRITON-TIMI 38 trial as having had TIMI major/minor bleeding events (1 patient was randomized to prasugrel but did not receive the study medication and 1 patient received clopidogrel). ${ }^{8}$ Patient flow is illustrated in Figure E1.

The patients undergoing CABG in TRITON-TIMI 38 consisted of a diverse group of patients as defined by multiple factors such as patient and procedural characteristics, acquired hemostatic defects, the use of open-label 
antiplatelet therapy before the procedure $(n=20)$, patients not receiving the study medication $(\mathrm{n}=56)$, or undergoing more than 1 operative cardiac procedure $(n=26)$, any of which can influence the type and number of blood product transfusions needed and the amount of blood loss. Thus, the population of interest for the present study was refined to include only the cohort of patients who underwent isolated CABG and who had received the study drug before the procedure. Of the 346 patients in this cohort, 173 received prasugrel, with data on blood transfusions available for 167 , and 173 received clopidogrel, with similar data available for 164 (Table E1). A description of the patient characteristics is listed in Table E1, as described previously. ${ }^{8}$

\section{Transfusions}

The predicted transfusion risk score by treatment group is illustrated in Figure E2. No difference was noted in the mean predicted risk score between cohorts (prasugrel 2.13 vs clopidogrel 2.31, $P=.53$ ). The transfusion risk score correlated highly $(P<.0001)$ with the total number of RBC units transfused for the 2 cohorts and approached a significant association with THC $(P=.058$; Figure 1$)$. No significant differences were seen in the mean number of RBC units or THCs transfused between the 2 cohorts.

Blood product transfusions by drug treatment are illustrated in Figure E3. The potential risk factors for platelet transfusions perioperatively for patients with follow-up data are listed in Table E2. The incidence of platelet transfusion was significantly greater statistically in the patients treated with prasugrel than in those treated with clopidogrel $(18.0 \%$ vs $9.8 \%, P=.033)$, as was the mean number of platelets units transfused ( $0.8 \mathrm{U}$ vs $0.4 \mathrm{U}$, respectively, $P=.047)$. However, no significant difference was seen for the transfusion of RBCs $(2.3 \mathrm{U}$ vs $1.9 \mathrm{U}, P=.59)$, plasma (1.1 U vs $0.7 \mathrm{U}, P=.072$ ), THCs (2.2 $\mathrm{U}$ vs 1.1 $\mathrm{U}, P=.069$ ), or TDE between the prasugrel and clopidogrel cohorts ( $4.4 \mathrm{U}$ vs $3.0 \mathrm{U}, P=.46$ ). The proportion of patients contributing to the calculation of TDE was not different between the 2 cohorts (prasugrel, 108/167 [64.7\%]; clopidogrel, 106/164 [64.6\%]).

The transfusion requirements for the patients analyzed from the day of the last dose of study drug to the day of CABG are listed in Table 1. The distribution for the days from the last dose by therapy did not differ between the prasugrel and clopidogrel cohorts $\left(P=.26, \chi^{2}\right.$ analysis). For patients with isolated CABG $(\mathrm{n}=422), 54 \%, 22 \%$, and $24 \%$ of patients (irrespective of whether transfusion information was available) underwent CABG surgery within 5, 6 to 7, or more than 7 days from the last dose of study drug, respectively. Aspirin use was $63 \%$ within 5 days of CABG and decreased to $39 \%$ within 1 day of CABG. No imbalances were observed between treatment arms. Dual antiplatelet therapy was resumed after surgery when deemed safe by the investigator, and no difference was
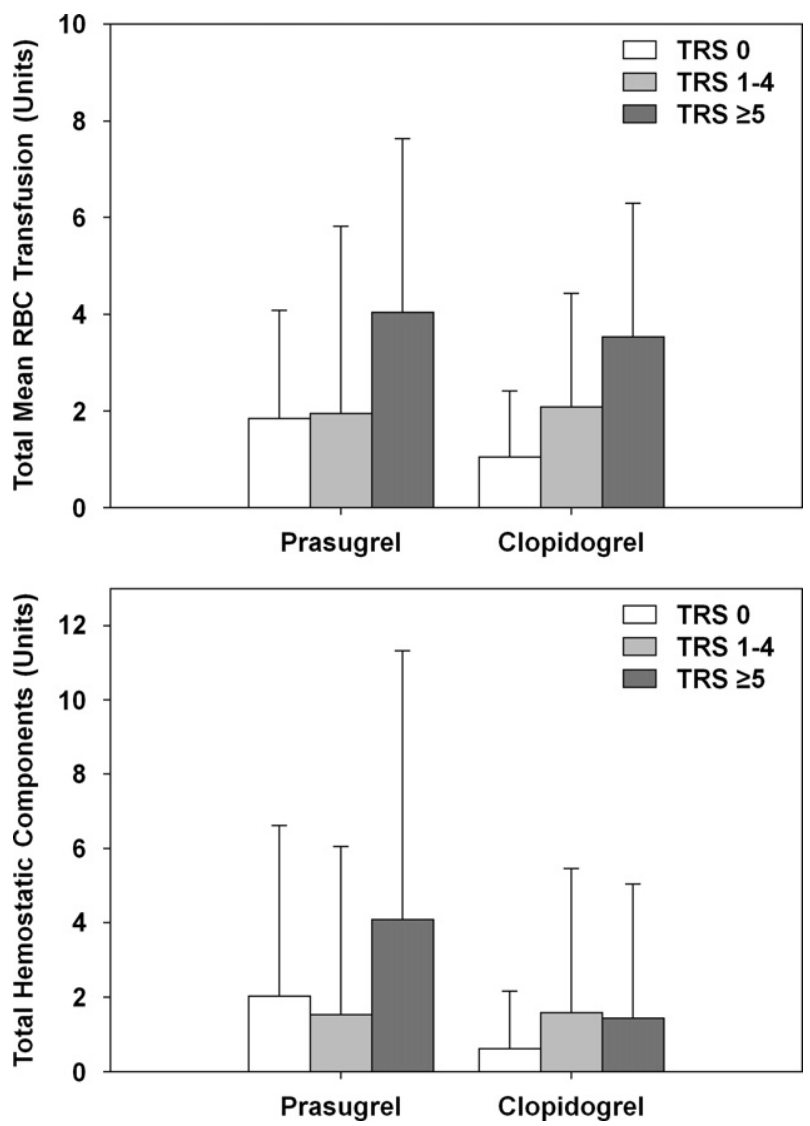

FIGURE 1. Transfusion risk score (TRS) association with total red blood cell $(R B C)$ transfusion (including whole blood and packed RBCs.) and total hemostatic components (including platelets, cryoprecipitate, and plasma). No significant differences were observed between cohorts.

observed between the treatment groups (Table E1). A significantly greater number of platelet units (mean $\pm \mathrm{SD}$; $0.95 \pm 2.84$ vs $0.25 \pm 1.14 \mathrm{U} ; P=.018$ ) were transfused postoperatively in the prasugrel cohort than in the clopidogrel cohort for those patients who underwent surgery within 5 days after withdrawal of the antiplatelet study drug. The TDE was not significantly different statistically between the study cohorts for any of the 3 intervals (Table 1). Additionally, a significantly greater mean cumulative chest tube blood loss was found at 12 hours in the prasugrel group compared with the clopidogrel group $(655 \pm 580 \mathrm{~mL}$ vs $503 \pm 378 \mathrm{~mL} ; P=.05$ ), with a high degree of variability in chest tube blood loss across each day from the last dose of study drug to CABG (Table 2). The TDE and THC correlated with chest tube blood loss at 12 hours, and the correlations were similar for both cohorts (Figure E4).

Postoperative complications and resource usage in association with CABG were analyzed according to the total RBC units transfused (Table 3). Eleven patients receiving prasugrel $(6.4 \%)$ and 4 patients receiving clopidogrel $(2.3 \%)$ underwent surgical re-exploration $(P=.07)$ for 
TABLE 1. Transfusion requirements from day of last dose of study drug to CABG

\begin{tabular}{|c|c|c|c|c|c|c|c|c|c|}
\hline \multirow[b]{2}{*}{ Variable } & \multicolumn{3}{|c|}{$\leq 5$ Days } & \multicolumn{3}{|c|}{ 6-7 Days } & \multicolumn{3}{|c|}{$>7$ Days } \\
\hline & $\begin{array}{l}\text { Prasugrel } \\
(\mathbf{n}=\mathbf{8 8})\end{array}$ & $\begin{array}{c}\text { Clopidogrel } \\
(\mathbf{n}=99)\end{array}$ & $\begin{array}{c}P \\
\text { value* }\end{array}$ & $\begin{array}{l}\text { Prasugrel } \\
(\mathbf{n}=\mathbf{3 4})\end{array}$ & $\begin{array}{c}\text { Clopidogrel } \\
(\mathrm{n}=\mathbf{4 1})\end{array}$ & $\begin{array}{c}P \\
\text { value* }\end{array}$ & $\begin{array}{l}\text { Prasugrel } \\
(\mathbf{n}=\mathbf{5 0})\end{array}$ & $\begin{array}{c}\text { Clopidogrel } \\
(\mathbf{n}=\mathbf{3 2})\end{array}$ & $\begin{array}{c}P \\
\text { value* }\end{array}$ \\
\hline \multicolumn{10}{|c|}{ Intraoperative } \\
\hline $\mathrm{RBCs}$ & $0.62 \pm 1.14$ & $0.84 \pm 1.14$ & .098 & $0.71 \pm 1.69$ & $0.59 \pm 1.04$ & .945 & $0.34 \pm 0.67$ & $0.79 \pm 1.91$ & .664 \\
\hline Platelets & $0.33 \pm 1.20$ & $0.22 \pm 1.13$ & .300 & $0 \pm 0$ & $0.05 \pm 0.32$ & .358 & $0.23 \pm 1.46$ & $0.29 \pm 1.19$ & .669 \\
\hline THCs & $0.77 \pm 2.24$ & $0.47 \pm 1.56$ & .267 & $0.09 \pm 0.38$ & $0.10 \pm 0.45$ & .887 & $0.30 \pm 1.49$ & $0.74 \pm 2.25$ & .485 \\
\hline TDE & $1.40 \pm 2.64$ & $1.30 \pm 1.98$ & .511 & $0.80 \pm 1.89$ & $0.69 \pm 1.08$ & .671 & $0.64 \pm 1.65$ & $1.53 \pm 3.87$ & .974 \\
\hline \multicolumn{10}{|c|}{ Postoperative } \\
\hline $\mathrm{RBCs}$ & $2.02 \pm 3.34$ & $1.27 \pm 1.77$ & .120 & $1.82 \pm 2.44$ & $0.92 \pm 1.60$ & .154 & $1.06 \pm 1.69$ & $0.84 \pm 1.24$ & .627 \\
\hline Platelets & $0.95 \pm 2.84$ & $0.25 \pm 1.14$ & .018 & $0.18 \pm 0.53$ & $0.08 \pm 0.48$ & .127 & $0.15 \pm 0.87$ & $0.23 \pm 1.09$ & .653 \\
\hline THCs & $2.31 \pm 5.81$ & $0.72 \pm 2.09$ & .155 & $1.48 \pm 3.13$ & $0.64 \pm 3.41$ & .006 & $0.75 \pm 1.80$ & $0.68 \pm 1.83$ & .858 \\
\hline TDE & $4.34 \pm 8.45$ & $1.99 \pm 3.28$ & .267 & $3.30 \pm 5.14$ & $1.56 \pm 4.71$ & .107 & $1.81 \pm 3.23$ & $1.52 \pm 2.71$ & .504 \\
\hline \multicolumn{10}{|l|}{ Total } \\
\hline RBCs & $2.64 \pm 3.91$ & $2.11 \pm 2.24$ & .594 & $2.53 \pm 2.96$ & $1.51 \pm 1.83$ & .297 & $1.40 \pm 1.94$ & $1.63 \pm 2.69$ & .847 \\
\hline Platelets & $1.25 \pm 3.25$ & $0.47 \pm 1.57$ & .039 & $0.18 \pm 0.53$ & $0.13 \pm 0.57$ & .322 & $0.38 \pm 1.67$ & $0.52 \pm 2.25$ & .793 \\
\hline THCs & $3.08 \pm 6.56$ & $1.18 \pm 2.51$ & .194 & $1.58 \pm 3.20$ & $0.74 \pm 3.42$ & .036 & $1.04 \pm 2.52$ & $1.42 \pm 3.88$ & .888 \\
\hline TDE & $5.71 \pm 9.45$ & $3.29 \pm 3.91$ & .556 & $4.11 \pm 5.64$ & $2.26 \pm 4.73$ & .349 & $2.44 \pm 3.80$ & $3.05 \pm 5.98$ & .548 \\
\hline
\end{tabular}

Data presented as mean \pm standard deviation. Number of subjects included those from last day of drug to surgery. $R B C s$, Red blood cells (packed RBCs, whole blood); TDE, total donor exposure (RBCs, platelets, cryoprecipitate, plasma); THCs, total hemostatic components (platelets, cryoprecipitate, plasma). $* P<.05$ (Kruskal-Wallis test).

bleeding. A bleeding source was identified in most (11/15) of the patients undergoing re-exploration. One patient in the prasugrel group died after surgical re-exploration; the death was not attributed to the study drug and was likely from the multiple co-morbidities. None of the 4 patients in the clopidogrel group died after surgical re-exploration. The length of the hospital and postoperative intensive care unit stays were similar between the prasugrel and clopidogrel groups, irrespective of the RBC transfusion outcomes.

The euroSCORE and STS risk-adjusted predicted mortality was calculable for 446 of 448 patients. In an analysis adjusted for the predicted risk of mortality (using

TABLE 2. Chest blood loss at 12 hours after CABG for patients who underwent isolated CABG in TRITON-TIMI 38 with follow-up data

\begin{tabular}{|c|c|c|c|}
\hline \multirow{2}{*}{$\begin{array}{l}\text { Interval from last study } \\
\text { drug dose to CABG (d) }\end{array}$} & \multicolumn{3}{|c|}{ Thienopyridine use before CABG } \\
\hline & Prasugrel & Clopidogrel & $P$ value* \\
\hline$<5$ & & & .114 \\
\hline Patients (n) & 65 & 75 & \\
\hline Blood loss (mL) & $679.1 \pm 617.6$ & $533.8 \pm 391.5$ & \\
\hline $6-7$ & & & .181 \\
\hline Patients (n) & 27 & 32 & \\
\hline Blood loss (mL) & $815.4 \pm 666.5$ & $462.8 \pm 366.9$ & \\
\hline$>7$ & & & .573 \\
\hline Patients (n) & 38 & 24 & \\
\hline Blood loss (mL) & $509.2 \pm 405.1$ & $463.6 \pm 365.5$ & \\
\hline Total & & & .050 \\
\hline Patients (n) & $131 \dagger$ & $132 \dagger$ & \\
\hline Blood loss (mL) & $655.0 \pm 579.8$ & $502.6 \pm 378.5$ & \\
\hline
\end{tabular}

Data presented as mean \pm standard deviation, unless otherwise noted. $C A B G$, Coronary artery bypass grafting; TRITON-TIMI 38 , Trial to Assess Improvement in Therapeutic Outcomes by Optimizing Platelet Inhibition With Prasugrel Thrombolysis in Myocardial Infarction 38 . ${ }^{*}$ Two-sided $P$ value, Kruskal-Wallis test. $\dagger$ One subject each receiving prasugrel and clopidogrel had chest tube blood loss data but no data on interval from last dose of study drug to CABG.
euroSCORE), TDE and THCs were not associated with increased mortality risk (all-cause death within 30 days after CABG, odds ratio, $1.06 ; 95 \%$ confidence interval, $0.98-1.14 ; P=.15$ for TDE, and odds ratio, $1.05 ; 95 \%$ confidence interval, $0.94-1.17 ; P=.40$ for THC; logistic regression analysis; Figure 2). Similar results were observed with the STS risk adjustment. As previously reported, ${ }^{8}$ treatment with prasugrel was independently associated with a reduction in mortality compared with treatment with clopidogrel $(1.2 \%$ vs $6.9 \%, P=.022)$. The causes of death, as determined by an adjudication committee, are listed in Table E3.

\section{DISCUSSION}

The present retrospective analysis included newly collected data from 346 patients who had undergone isolated CABG after withdrawal from either prasugrel $(n=173)$ or clopidogrel $(\mathrm{n}=173)$ during the TRITON-TIMI 38 study. A companion analysis demonstrated that despite an increase in observed bleeding, prasugrel was associated with a lower rate of death in ACS patients undergoing $\mathrm{CABG}^{8}{ }^{8}$ In the present analysis, treatment with prasugrel was associated with significantly greater chest tube drainage at 12 hours postoperatively, and a greater percentage of patients received platelet transfusions and had a greater mean number of platelets transfused (Figure E2). The THCs transfused was not different between the 2 cohorts. The platelet transfusion differences were predominately driven by differences in the platelet transfusion rates within the subgroup of patients who underwent CABG within 5 days of discontinuing their antiplatelet therapy (Table 1).

The percentage of patients who received platelet transfusions in the 2 cohorts (18\% vs $9.8 \%$ for prasugrel vs clopidogrel, respectively; $P=.033$ ) in the present 
TABLE 3. Surgical re-exploration and resource usage after CABG according to total RBC units

\begin{tabular}{|c|c|c|c|c|c|c|}
\hline & \multicolumn{6}{|c|}{ Total RBC units transfused during CABG } \\
\hline & \multicolumn{2}{|c|}{ None } & \multicolumn{2}{|c|}{$>0-2 \mathrm{U}$} & \multicolumn{2}{|c|}{$>\mathbf{2} \mathbf{U}$} \\
\hline & Prasugrel & Clopidogrel & Prasugrel & Clopidogrel & Prasugrel & Clopidogrel \\
\hline $\begin{array}{l}\text { Surgical re-exploration for } \\
\text { bleeding }\end{array}$ & $0(0)(\mathrm{n}=64)$ & $1(1.6)(\mathrm{n}=62)$ & $0(0)(\mathrm{n}=51)$ & $0(0)(\mathrm{n}=56)$ & $11(21.2)(\mathrm{n}=52)$ & $3(6.7)(\mathrm{n}=45)$ \\
\hline $\begin{array}{l}\text { Bleeding source identified by } \\
\text { surgical re-exploration* }\end{array}$ & $\mathrm{NA}(\mathrm{n}=0)$ & $1(100)(\mathrm{n}=1)$ & NA $(n=0)$ & $\mathrm{NA}(\mathrm{n}=0)$ & $8(72.7 \%)(n=11)$ & $2(66.7)(\mathrm{n}=3)$ \\
\hline Hospital stay (d) & $12 \pm 9(\mathrm{n}=63)$ & $13 \pm 15(\mathrm{n}=62)$ & $11 \pm 5(\mathrm{n}=51)$ & $10 \pm 4(\mathrm{n}=56)$ & $13 \pm 11(\mathrm{n}=52)$ & $14 \pm 9(\mathrm{n}=45)$ \\
\hline Postoperative ICU stay (h) & $64 \pm 109(\mathrm{n}=59)$ & $60 \pm 102(\mathrm{n}=59)$ & $51 \pm 42(\mathrm{n}=49)$ & $48 \pm 34(\mathrm{n}=54)$ & $101 \pm 192(\mathrm{n}=52)$ & $98 \pm 113(n=44)$ \\
\hline
\end{tabular}

Data presented as n (\%), with $\mathrm{n}$ in parentheses, number of subjects with nonmissing values for each category, or mean \pm standard deviation. $C A B G$, Coronary artery bypass grafting; $I C U$, intensive care unit; $N A$, not allowed because denominator was 0 (ie, no patients in category underwent surgical re-exploration); $R B C$, red blood cell. * Denominator consisted of patients who underwent surgical re-exploration.

analysis were in line with that in the STS database and others' reported experience. For example, patients undergoing isolated CABG reported to the STS database (January to December 2010) received platelet transfusions in $24 \%$ of cases; only $14 \%$ of all isolated CABG patients were treated with an antiplatelet adenosine diphosphate (ADP) inhibitor (prasugrel or clopidogrel) within 5 days of the CABG procedure (Society of Thoracic Surgeons, 2010, Chicago, Ill, unpublished data, April 2011). A recent publication from an STS database cohort of 82,446 patients undergoing isolated
CABG surgery at 408 sites reported highly variable platelet transfusion rates $(0.4 \%-90 \%$ of patients) among institutions, with the 25th to 50th percentiles for the probability of platelet transfusions showing adjusted true platelet transfusion rates of $14.7 \%$ to $25.3 \%$. $^{9}$ These rates are comparable to the platelet transfusion outcomes in the 2 cohorts in our analysis. We interpret these data to indicate that antiplatelet therapy with ADP inhibitors within 5 days of isolated CABG procedures, although associated with increased postoperative chest tube losses and associated increased platelet


FIGURE 2. Predicted probability of mortality as function of total donor exposure and function of total hemostatic components. In analyses adjusted for predicted risk of mortality (using European System for Cardiac Operative Risk Evaluation Score [euroSCORE]), increased mortality risk (all-cause death within 30 days after coronary artery bypass grafting [CABG]) was not associated with total donor exposure (odds ratio, 1.06; 95\% confidence interval, $0.98-1.14 ; P=.15$; logistic regression analysis) nor total hemostatic components (odds ratio, $1.05 ; 95 \%$ confidence interval, $0.94-1.17 ; P=.40 ;$ logistic regression analysis). Prasugrel was independently associated with reduction in mortality (prasugrel, $1.2 \%$; clopidogrel, $6.9 \% ; P=.022$ ). Total donor exposure included red blood cells, platelets, cryoprecipitate, and plasma; total hemostatic components included platelets, cryoprecipitate, and plasma. 
transfusions, is not associated with an untoward need for blood component transfusion support.

We also observed increased postoperative bleeding in patients who received prasugrel before surgery (Table 2). This finding of increased bleeding and platelet transfusion with a short preoperative interval is consistent with the recommendations in the labeled indications for both clopidogrel ${ }^{10}$ and prasugrel. ${ }^{11}$ For patients undergoing urgent CABG in whom significant antiplatelet effect remains, platelet therapy should be considered appropriate and therapeutic in the setting of clinical bleeding in the perioperative interval. However, the efficacy of platelet transfusion in this setting requires additional study, because transfused platelets can be inhibited by ADP inhibitor primary metabolites. Agents that augment platelet function or that activate platelets independent of an ADP/P2Y12 receptor mechanisms (ie, by way of thrombin) might have a role in the management of refractory and life-threatening bleeding when these agents are administered. The current American College of Cardiology Foundation/American Heart Association guidelines recommend discontinuation of ADP antagonists at least 5 days before surgery for clopidogrel and at least 7 days before surgery for prasugrel to minimize bleeding complications. ${ }^{12}$

Although platelet transfusions and overall hemostatic component therapy were increased in the prasugrel cohort, neither RBC transfusions nor total blood components transfused (TDE) were significantly different statistically between the prasugrel and clopidogrel cohorts in the 3 intervals analyzed (Table 1). Although an analysis of patients who received more than $2 \mathrm{U}$ of RBCs indicated that a greater percentage of patients who received prasugrel $(21 \%)$ underwent surgical re-exploration for bleeding compared with those receiving clopidogrel $(7 \%)$, most patients (10/14 for these patients and 11/15 for all patients) who underwent re-exploration had a surgical bleeding source identified (Table 3). Despite increased postoperative chest tube losses, platelet transfusions, and observed re-exploration rates, overall mortality was lower in the prasugrel cohort.

Our analysis had several potential limitations. The data were collected after completion of the primary clinical trial, which was designed to evaluate the efficacy of prasugrel versus clopidogrel in patients undergoing PCI. The data on any differences in blood transfusion requirements should be evaluated in the context of the wide variability in the transfusion of blood products, independent of case mix, among patients who undergo CABG surgery. ${ }^{9,13}$ Per protocol, specific blood component therapy for bleeding was at the discretion of the investigator and should be interpreted in the context of the characteristics and parameters listed in Tables E1 and E2. Even with a larger powered study, any potential differences in total allogeneic donor exposures (Figure E2) would be in the context of improved mortality in the cohort receiving prasugrel therapy. Finally, the nonrandomized nature of the decision to perform $\mathrm{CABG}$ and the timing of study drug withdrawal had the potential to introduce selection bias that could have confounded the comparison between prasugrel and clopidogrel.

\section{CONCLUSIONS}

For the TRITON-TIMI 38 patients who underwent CABG, RBC transfusion, THCs, and TDE were not significantly different statistically between the cohorts treated with prasugrel or clopidogrel. Only the incidence and number of platelet transfusions were significantly greater statistically in the prasugrel cohort than in the clopidogrel cohort, consistent with the increases in 12-hour postoperative chest tube drainage in the prasugrel cohort. Although the postoperative chest tube blood loss and platelet transfusions were significantly increased in the prasugrel cohort, the use of prasugrel was not associated with increased RBC transfusions, THC transfusions, or TDE.

The authors thank Ms Ingrid Liu for statistical support.

\section{References}

1. Yusuf S, Zhao F, Mehta SR, Chrolavicius S, Tognoni G, Fox KK, et al. Effects of clopidogrel in addition to aspirin in patients with acute coronary syndromes without ST-segment elevation. $N$ Engl J Med. 2001;345:494-502. erratum, $N$ Engl J Med 2001;345:1506, 1716.

2. Wiviott SD, Braunwald E, McCabe CH, Montalescot G, Ruzyllo W, Gottlieb S, et al. Prasugrel versus clopidogrel in patients with acute coronary syndromes. $N$ Engl J Med. 2007;357:2001-15.

3. Wiviott SD, Antman EM, Gibson CM, Montalescot G, Riesmeyer J, Weerakkody G, et al. Evaluation of prasugrel compared with clopidogrel in patients with acute coronary syndromes: design and rationale for the TRial to assess Improvement in Therapeutic Outcomes by optimizing platelet InhibitioN with prasugrel Thrombolysis In Myocardial Infarction 38 (TRITON-TIMI 38). Am Heart J. 2006;152:627-35.

4. Wiviott SD, Braunwald E, Murphy SA, Antman EA. Intensive antiplatelet therapy for reduction of ischaemic events-authors' reply. Lancet. 2008;372:532.

5. Magovern JA, Sakert T, Benckart DH, Burkholder JA, Liebler GA, Magovern GJ Sr, et al. A model for predicting transfusion after coronary artery bypass grafting. Ann Thorac Surg. 1996;61:27-32.

6. Anderson RP. First publications from the Society of Thoracic Surgeons National Database. Ann Thorac Surg. 1994;57:6-7.

7. Nashef SA, Roques F, Michel P, Gauducheau E, Lemeshow S, Salamon R. European system for cardiac operative risk evaluation (euroSCORE). Eur J Cardiothorac Surg. 1999;16:9-13.

8. Smith PK, Goodnough LT, Levy JH, Poston RS, Short MA, Weerakkody GJ, et al. Mortality benefit with prasurgrel in the TRITON-TIMI 38 coronary artery bypass grafting cohort: risk-adjusted retrospective data analysis. J Am Coll Card. 2012;60:388-96.

9. Bennett-Guerrero E, Zhao Y, O'Brien SM, Ferguson TB Jr, Peterson ED, Gammie JS, et al. Variation in use of blood transfusion in coronary artery bypass graft surgery. JAMA. 2010;304:1568-75.

10. Plavix. Prescribing information. Available from: http://products.sanofi.us/ PLAVIX/PLAVIX.html. Accessed December 4, 2011.

11. Effient. Prescribing information. Available from: http://pi.lilly.com/us/effient. pdf. Accessed December 4, 2011

12. Wright RS, Anderson JL, Adams CD, Bridges CR, Casey DE Jr, Ettinger SM, et al. 2011 ACCF/AHA focused update of the Guidelines for the Management of Patients with Unstable Angina/Non-ST-Elevation Myocardial Infarction (updating the 2007 guideline): a report of the American College of Cardiology Foundation/American Heart Association Task Force on Practice Guidelines developed in collaboration with the American College of Emergency Physicians, Society for Cardiovascular Angiography and Interventions, and Society of Thoracic Surgeons. J Am Coll Cardiol. 2011;57:1920-59.

13. Shander AS, Goodnough LT. Blood transfusion as a quality indicator in cardiac surgery (editorial). JAMA. 2010;304:1610-1. 


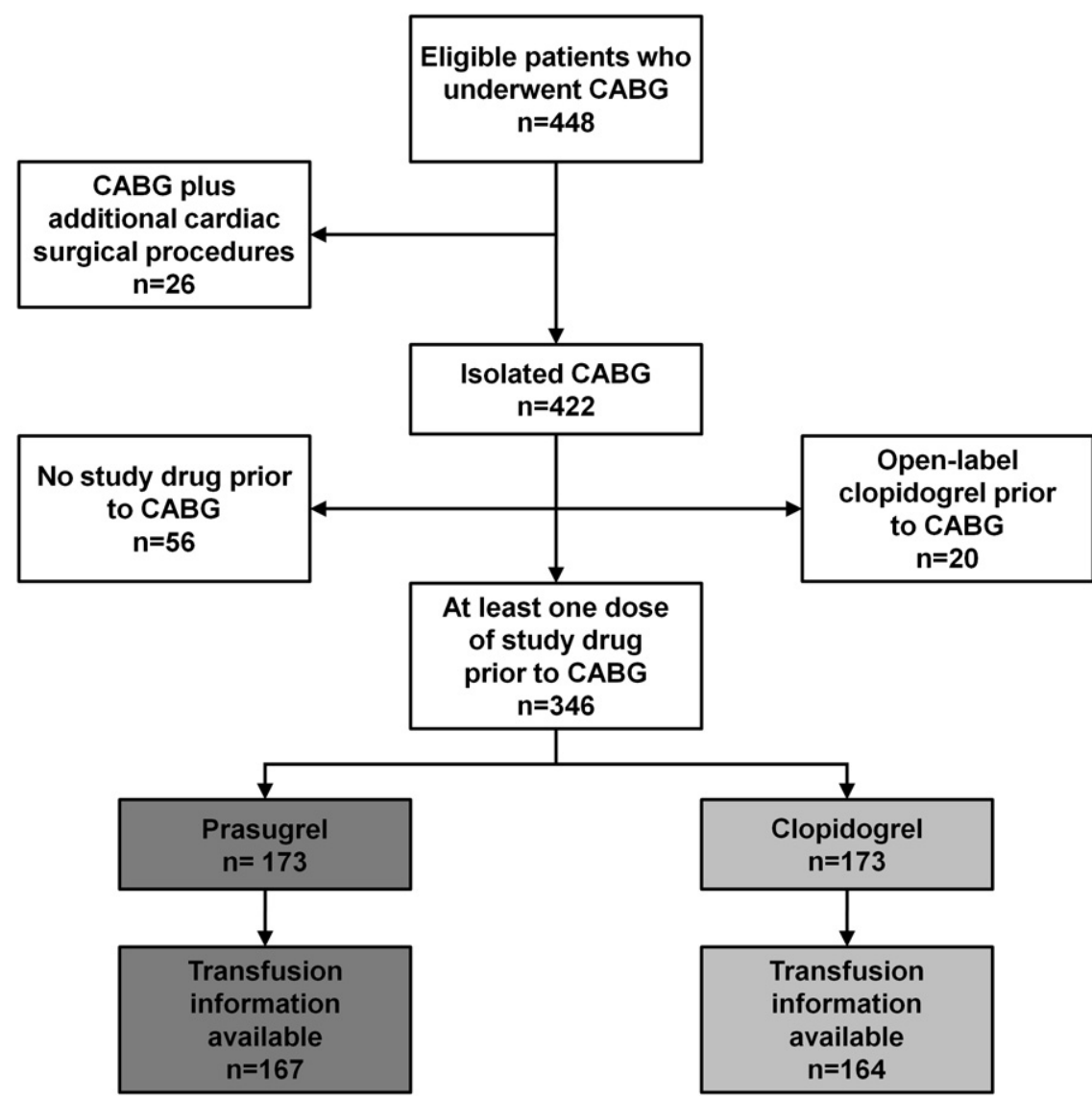

FIGURE E1. Flow diagram for Trial to Assess Improvement in Therapeutic Outcomes by Optimizing Platelet Inhibition With Prasugrel Thrombolysis in Myocardial Infarction 38 (TRITON-TIMI 38) patients who underwent coronary artery bypass grafting (CABG).

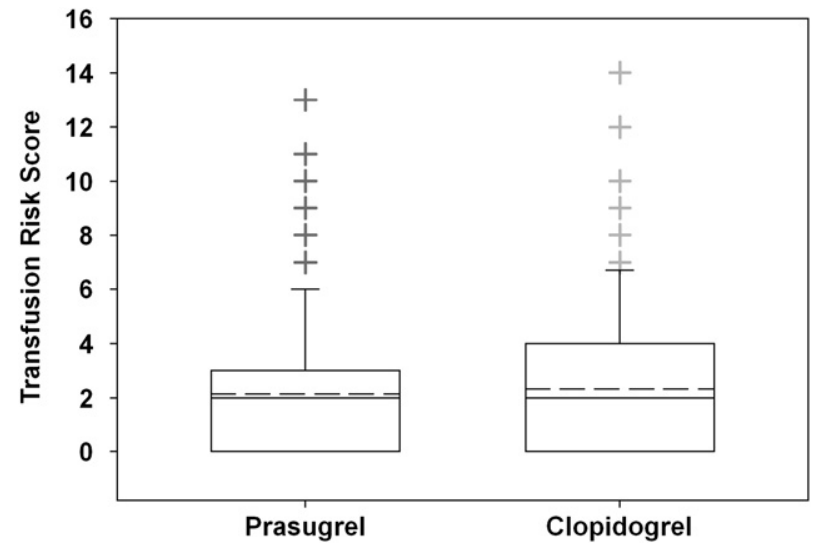

FIGURE E2. Predicted transfusion risk score by treatment group. No statistically significant difference noted in mean predicted transfusion risk score between cohorts (prasugrel, 2.13 vs clopidogrel, $2.31 ; P=.53$; 2 -sided $P$ value, analysis of variance). Solid lines represent medians, dashed lines, means; boxes, 25th to 75th percentiles; upper whisker, 90th percentile; plus signs, represent values greater than 90th percentile.

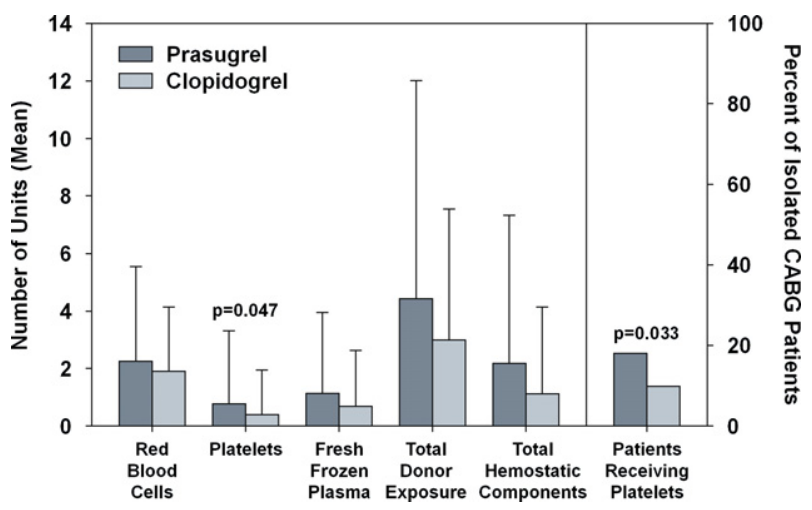

FIGURE E3. Mean number of blood product units transfused by treatment group. Number of platelet units transfused and percentage of patients receiving platelet transfusions were statistically greater in prasugrel cohort (red blood cells included packed red blood cells and whole blood; total hemostatic components included platelets, cryoprecipitate, plasma; total donor exposure included red blood cells, platelets, cryoprecipitate, and plasma). $C A B G$, Coronary artery bypass grafting. 

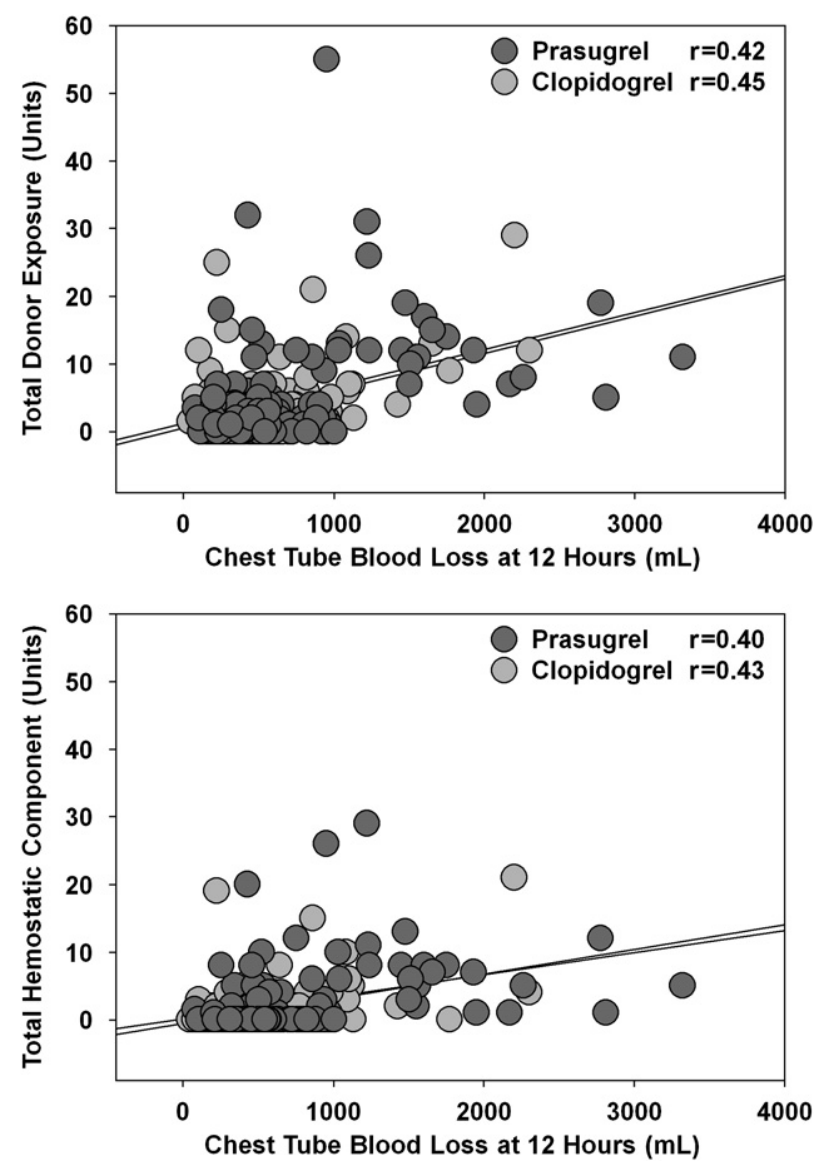

FIGURE E4. Correlation of total donor exposure and total hemostatic components with chest tube blood loss at 12 hours. Correlations were similar for both cohorts for both analyses. Total donor exposure included red blood cells, platelets, cryoprecipitate, and plasma; total hemostatic components included platelets, cryoprecipitate, and plasma. 
TABLE E1. Demographics and clinical characteristics for 2 patient cohorts

\begin{tabular}{|c|c|c|c|}
\hline Variable & Prasugrel $(n=173)$ & Clopidogrel $(\mathbf{n}=173)$ & $P$ value* \\
\hline \multicolumn{4}{|l|}{ Demographics } \\
\hline Age (y) & $61.1(9.3)$ & $60.9(10.2)$ & .856 \\
\hline Age $\geq 75$ y & $14(8.1)$ & $12(6.9)$ & .683 \\
\hline Men & $130(75.1)$ & $135(78.0)$ & .526 \\
\hline Weight $<60 \mathrm{~kg}$ & $13(7.7)$ & $10(5.9)$ & .527 \\
\hline \multicolumn{4}{|l|}{ Medical history } \\
\hline Cerebrovascular disease & $14(8.1)$ & $13(7.5)$ & .841 \\
\hline Hypertension & $115(66.5)$ & $107(61.9)$ & .370 \\
\hline Diabetes mellitus & $51(29.5)$ & $47(27.2)$ & .633 \\
\hline Cardiovascular surgery & $3(1.7)$ & $7(4.1)$ & .196 \\
\hline Chronic pulmonary disease & $8(4.6)$ & $20(11.6)$ & .017 \\
\hline Heart failure & $21(12.2)$ & $16(9.3)$ & .384 \\
\hline Ejection fraction $\leq 30 \%$ & $2(1.2)$ & $8(4.6)$ & .062 \\
\hline Peripheral vascular disease & $13(7.5)$ & $11(6.4)$ & .672 \\
\hline MI within $24 \mathrm{~h}$ before $\mathrm{CABG}$ & $6(3.5)$ & $8(4.6)$ & .585 \\
\hline PCI stent during TRITON index event & $145(83.8)$ & $137(79.2)$ & .268 \\
\hline Median interval from enrollment and/or stent to CABG (d) & 111.4 & 88.2 & .183 \\
\hline \multicolumn{4}{|l|}{ Procedural characteristics } \\
\hline PCI within $6 \mathrm{~h}$ of CABG & $12(6.9)$ & $6(3.5)$ & .146 \\
\hline CABG during TRITON index event & $8(4.62)$ & $7(4.05)$ & .792 \\
\hline CABG status $(\%)$ & & & .931 \\
\hline Elective & 84.4 & 85.0 & \\
\hline Urgent & 13.9 & 12.7 & \\
\hline Emergent or salvage & 1.7 & 2.3 & \\
\hline Intra-aortic balloon pump use & 7.6 & 11.8 & .194 \\
\hline Diseased vessels (n) & & & .371 \\
\hline 1 & $26(15.0)$ & $24(13.9)$ & \\
\hline 2 & $108(62.4)$ & $105(60.7)$ & \\
\hline 3 & $36(20.8)$ & $35(20.2)$ & \\
\hline Reoperation (previous CABG before enrollment) & $3(1.7)$ & $7(4.0)$ & .199 \\
\hline \multicolumn{4}{|l|}{ Antifibrinolytic therapy } \\
\hline Yes & $67(38.73)$ & $61(35.26)$ & .504 \\
\hline No & $106(61.27)$ & $112(64.74)$ & \\
\hline DAPT before CABG (interval from last antiplatelet dose to CABG [d]) & & & .257 \\
\hline 0 & $16(9.2)$ & $14(8.1)$ & \\
\hline 1 & $15(8.7)$ & $13(7.5)$ & \\
\hline 2 & $12(6.9)$ & $17(9.8)$ & \\
\hline 3 & $12(6.9)$ & $10(5.8)$ & \\
\hline 4 & $9(5.2)$ & $11(6.4)$ & \\
\hline 5 & $24(13.9)$ & $34(19.7)$ & \\
\hline 6 & $21(12.1)$ & $16(9.2)$ & \\
\hline 7 & $13(7.5)$ & $25(14.5)$ & \\
\hline $8-14$ & $36(20.8)$ & $20(11.6)$ & \\
\hline$>14$ & $14(8.1)$ & $12(6.9)$ & \\
\hline Resumed DAPT (open label or study drug) after CABG & $129(74.57)$ & $122(70.52)$ & 399 \\
\hline
\end{tabular}

Data presented as n (\%), unless indicated otherwise. $C A B G$, Coronary artery bypass grafting; $D A P T$, Dual antiplatelet therapy; $M I$, myocardial infarction, $P C I$, percutaneous coronary intervention, TRITON, Trial to Assess Improvement in Therapeutic Outcomes by Optimizing Platelet Inhibition With Prasugrel Thrombolysis in Myocardial Infarction 38. $* P$ values calculated using Pearson's $\chi^{2}$ test (categorical variables), Fisher's exact test, or Kruskal-Wallis test and analysis of variance (continuous variables). 
TABLE E2. Parameters relevant to platelet transfusion decisions

\begin{tabular}{|c|c|c|c|c|c|c|c|c|c|}
\hline & \multicolumn{8}{|c|}{ Platelets transfused during surgery* } & \multirow[b]{4}{*}{$P$ value $f$} \\
\hline & \multicolumn{4}{|c|}{ No } & \multicolumn{4}{|c|}{ Yes } & \\
\hline & \multicolumn{2}{|c|}{ Prasugrel $(n=144)$} & \multicolumn{2}{|c|}{ Clopidogrel $(\mathbf{n}=155)$} & \multicolumn{2}{|c|}{ Prasugrel $(n=29)$} & \multicolumn{2}{|c|}{ Clopidogrel $(n=16)$} & \\
\hline & Patients (n) & Mean \pm SD & Patients (n) & Mean \pm SD & Patients (n) & Mean \pm SD & Patients (n) & Mean \pm SD & \\
\hline Platelet count $\left(10^{3} / \mathrm{mm}^{3}\right)$ & 126 & $237 \pm 72.9$ & 146 & $235 \pm 81.7$ & 27 & $216 \pm 66.6$ & 16 & $274 \pm 118.8$ & .830 \\
\hline Total pump time (min) & 100 & $80 \pm 36.3$ & 122 & $78 \pm 32.8$ & 24 & $103 \pm 63.5$ & 14 & $81 \pm 25.4$ & .036 \\
\hline \multicolumn{10}{|l|}{ Chest tube blood loss (mL) } \\
\hline $8 \mathrm{~h}$ & 104 & $399 \pm 402.6$ & 118 & $370 \pm 257.9$ & 26 & $991 \pm 597.8$ & 15 & $707 \pm 503.1$ & $<.001$ \\
\hline $12 \mathrm{~h}$ & 105 & $516 \pm 455.0$ & 117 & $437 \pm 265.6$ & 26 & $1218 \pm 689.9$ & 15 & $1014 \pm 663.1$ & $<.001$ \\
\hline $24 \mathrm{~h}$ & 105 & $728 \pm 554.2$ & 122 & $630 \pm 374.8$ & 26 & $1601 \pm 859.5$ & 15 & $1370 \pm 773.5$ & $<.001$ \\
\hline At discontinuation & 109 & $907 \pm 794.4$ & 125 & $831 \pm 623.1$ & 26 & $2627 \pm 2342$ & 15 & $1893 \pm 1418$ & $<.001$ \\
\hline
\end{tabular}

Data presented as mean \pm standard deviation, unless otherwise noted. *Yes indicates $\geq 1 \mathrm{U}$ of platelets transfused. $\dagger P$ value for risk factor based on logistic regression, with response $=0,>0 \mathrm{U}$ of platelets; independent variables: drug (prasugrel or clopidogrel) and baseline risk factor of interest.

TABLE E3. Causes of death

\begin{tabular}{lcc}
\hline \multicolumn{1}{c}{ Cause of death } & \multicolumn{1}{c}{ Treatment cohort } \\
\cline { 2 - 3 } & Prasugrel & Clopidogrel \\
\hline Congestive heart failure/cardiogenic shock & 2 & 4 \\
Myocardial Infarction & 1 \\
Infection & 1 & 1 \\
Other tamponade & - & - \\
Other gastrointestinal & - & 1 \\
Accidental/trauma & - & 1 \\
Related to revascularization (CABG or PCI) & - & 5 \\
Malignancy & - & 1 \\
Sudden or unwitnessed death & - & 1 \\
\hline
\end{tabular}

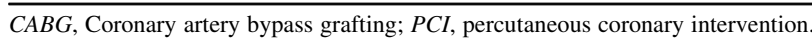

